\title{
Insights into the genome structure and copy-number variation of Eimeria tenella
}

\author{
Lik-Sin Lim ${ }^{1,2}$, Yea-Ling Tay ${ }^{1,2}$, Halimah Alias ${ }^{1}$, Kiew-Lian Wan ${ }^{1,2^{*}}$ and Paul H Dear ${ }^{3^{*}}$
}

\begin{abstract}
Background: Eimeria is a genus of parasites in the same phylum (Apicomplexa) as human parasites such as Toxoplasma, Cryptosporidium and the malaria parasite Plasmodium. As an apicomplexan whose life-cycle involves a single host, Eimeria is a convenient model for understanding this group of organisms. Although the genomes of the Apicomplexa are diverse, that of Eimeria is unique in being composed of large alternating blocks of sequence with very different characteristics - an arrangement seen in no other organism. This arrangement has impeded efforts to fully sequence the genome of Eimeria, which remains the last of the major apicomplexans to be fully analyzed. In order to increase the value of the genome sequence data and aid in the effort to gain a better understanding of the Eimeria tenella genome, we constructed a whole genome map for the parasite.

Results: A total of 1245 contigs representing $70.0 \%$ of the whole genome assembly sequences (Wellcome Trust Sanger Institute) were selected and subjected to marker selection. Subsequently, 2482 HAPPY markers were developed and typed. Of these, 795 were considered as usable markers, and utilized in the construction of a HAPPY map. Markers developed from chromosomally-assigned genes were then integrated into the HAPPY map and this aided the assignment of a number of linkage groups to their respective chromosomes. BAC-end sequences and contigs from whole genome sequencing were also integrated to improve and validate the HAPPY map. This resulted in an integrated HAPPY map consisting of 60 linkage groups that covers approximately half of the estimated $60 \mathrm{Mb}$ genome. Further analysis suggests that the segmental organization first seen in Chromosome 1 is present throughout the genome, with repeat-poor $(P)$ regions alternating with repeat-rich $(R)$ regions. Evidence of copy-number variation between strains was also uncovered.

Conclusions: This paper describes the application of a whole genome mapping method to improve the assembly of the genome of $E$. tenella from shotgun data, and to help reveal its overall structure. A preliminary assessment of copy-number variation (extra or missing copies of genomic segments) between strains of E. tenella was also carried out. The emerging picture is of a very unusual genome architecture displaying inter-strain copy-number variation. We suggest that these features may be related to the known ability of this parasite to rapidly develop drug resistance.
\end{abstract}

\section{Background}

The phylum Apicomplexa contains a diverse range of parasites including Plasmodium, Cryptosporidium, Babesia, Toxoplasma and others that cause disease in both humans and animals. The genomes of several apicomplexans have been extensively studied [1-5] in an

\footnotetext{
* Correspondence: klwan@ukm.my; phd@mrc-Imb.cam.ac.uk

${ }^{1}$ School of Biosciences and Biotechnology, Faculty of Science and Technology, Universiti Kebangsaan Malaysia, 43600, UKM Bangi, Selangor DE, Malaysia

${ }^{3}$ MRC Laboratory of Molecular Biology, Hills Road, Cambridge CB2 OQH, United Kingdom

Full list of author information is available at the end of the article
}

attempt to understand these organisms and to gain insights into potential new methods of control and treatment. Although a few common features have emerged, the apicomplexan genomes studied to date have presented a remarkable diversity of genomic organization and gene content, with each genome having its own unique, and often unusual, characteristics. The genus Eimeria, belonging to the family Eimeriidae within the order Eucoccidiorida (commonly known as the coccidians) represents the last major group of apicomplexans to be analyzed in detail. Because it is homoxenous, Eimeria can be propagated to high numbers in a single life-cycle with relative ease, making it a potential model

\section{Biomed Central}


for aspects of apicomplexan biology that are difficult to study in other genera.

Eimeria is also significant in its own right, as the causative agent of the intestinal disease coccidiosis in poultry [6]. Eimeria species are both site- and host-specific, and are transmitted through the ingestion of sporulated oocysts - resistant, hardy, thick-walled spores that contain infective sporozoites [7]. Seven species infect chickens and Eimeria tenella is among the most pathogenic [8] causing weight loss, reduced feed efficiency, reduced egg production and death. The total loss including the costs of control and prevention worldwide is estimated at around USD2.4 billion per annum, making this one of the most economically important diseases of domestic livestock [9].

The desire for a better understanding of the parasite and its interaction with the chicken, and a need for better disease control, had driven the E. tenella genome sequencing project [10]. A draft genome assembly (released by the Wellcome Trust Sanger Institute in May 2007) of 8.3-fold sequence coverage contains 4707 contigs, ranging from thousands to half a million bases in length. The total size of these contigs is $47 \mathrm{Mb}$ corresponding to $\sim 78 \%$ of the estimated $60 \mathrm{Mb}$ genome. A further assembly, incorporating second-generation sequencing data, was produced in 2010, but much of the genome remains unrepresented by large contigs.

The completion of eukaryotic genome sequencing projects depends heavily on having good genome maps to position contigs, give information on large-scale genome structure, and reveal errors in sequence assembly [11-13]. Such a map has hitherto been lacking in the case of E. tenella. The available genetic linkage map, which relies on polymorphic loci, is low in resolution and does not reflect the genome physically [14]. Furthermore, large insert clones are difficult to produce for Eimeria species as they are often unstable due to repetitive sequences [15], eliminating an effective method for physical mapping.

HAPPY mapping [16] is an in vitro physical mapping technique which analyses markers' co-segregation amongst a pool of sub-genomic samples (each containing an approximately haploid amount of randomly sheared DNA). Markers that are close together tend to cosegregate strongly amongst the aliquots. Each marker will be typed by PCR to detect its presence in each aliquot and the probability of linkage between two markers is given as a logarithm of odds (LOD) score. Based on the LOD scores, a genome map can be constructed. This technique was successful in assisting the assembly of $E$. tenella chromosomes 1 and 2 with individual maps constructed respectively by Ling et al. [17] and Paul H. Dear (unpublished data).

Mapping also provides an opportunity to examine genomic variation. Comparisons between different strains of
E. tenella have found only limited sequence variation $[18,19]$. Recent studies in several species (including human) have shown that structural variation (duplication or rearrangement) contributes more diversity than sequence variation [20], but there have been no efforts to look into structural variations in Eimeria or other Apicomplexa. HAPPY mapping can be extended to allow molecular copy-number counting (MCC), determining copy-numbers by counting the molecules present [21]. MCC has been used to screen variations in copy-number due to its speed, flexibility and sensitivity [22-24].

As HAPPY maps for E. tenella chromosomes 1 and 2 have previously been constructed, we demonstrate in this study the construction of an integrated map for the remainder of the genome in order to give an overview of genome organization and to aid sequence assembly. We managed to improve and validate the map by integrating BAC-end and contig sequences. We find that the striking segmentation of the genome into feature-poor (P) and feature-rich (R) regions (previously noted on Chromosome 1) is present throughout the genome, and that the $\mathrm{P}$ - and R-segments correspond to unique and non-unique regions, respectively. Investigation of copynumber variation using MCC has highlighted further structural perspectives on the E. tenella genome.

\section{Results}

\section{Whole genome HAPPY map of Eimeria tenella}

Markers were generated from the draft genome assembly (released in May 2007) obtained from the Wellcome Trust Sanger Institute (WTSI). Contigs similar to Chromosome 1 [17] and 2 (unpublished data) were filtered prior to marker generation. A total of 1245 of the largest contigs, representing $70 \%$ of the assembled sequence, were selected. In total, 2482 markers were developed and typed. Of these, 576 (23.2\%) markers failed to show amplification and therefore were discarded. A total of 914 (36.8\%) multi-copy markers (recognized by an excess number of aliquots scoring positive for the marker) were also set aside while 231 (9.3\%) low-copy markers were further analyzed to screen for significant linkage. Overall, a total of 761 (30.7\%) good markers were obtained (details in Additional file 1). This low proportion of usable markers was due largely to the presence of sequences which, though unique in the assembly, were present in multiple copies in the genome. There are 301 contigs that carry only non-multicopy markers (unique contigs) while 279 contigs contain only multi-copy markers (multi-copy contigs). In these latter cases, up to nine unsuccessful attempts were made to identify unique sequences elsewhere in the contigs. The multi-copy contigs are up to $196 \mathrm{~kb}$ in length. 
Together with 34 low-copy markers which showed significant linkages, a total of 795 markers were used for the construction of the HAPPY map. Of these, 664 fell into 67 linkage groups of three or more markers, while 18 formed pairs of linked markers, at LOD $\geq 6$ (odds of better than one million to one in favor of linkage), leaving 113 markers as singletons (details in Additional file 2 ). The relative physical size of the map was calculated to be $27.3 \mathrm{Mb}$, or almost half of the estimated $60 \mathrm{Mb}$ genome. The HAPPY map linked 454 contigs with a total size of $18,224,408 \mathrm{bp}$, meaning that approximately $9 \mathrm{Mb}$ of gaps between contigs were bridged by HAPPY linkages alone.

\section{Data integration}

To produce a more comprehensive map, we integrated BAC-end sequences. The BAC clones of E. tenella have been end-sequenced in the WGS project, but were not used in the 2007 sequence assembly. We have also noticed that linkages by BAC clones alone can be unreliable, since many BAC-end sequences do not map uniquely to the genome, and some BACs show possible size discrepancies when both ends map to a single contig. However, the combination of BAC data with HAPPY map data is more robust.

Analysis of 9567 BAC-end sequences found a total of 2514 clones that aligned to different contigs of the assembly, including 277 linkages supported by at least two BACs (details in Additional file 3). Five new linkages (that is, linkages which had not already been made by HAPPY data alone) were identified by these clones, between the HAPPY linkage groups and large contigs that do not have any good markers. Most of the HAPPY linkages were supported by at least one BAC clone. Furthermore, 14 groups of multi-copy contigs were linked by BAC clones. The number of BAC clones that fall within these multi-copy contig groups often exceeds the number of clones within the HAPPY linkage group. The total size of all the contigs within the group ranged from about $100 \mathrm{~kb}$ to $400 \mathrm{~kb}$. Two groups were found to have one of their end-markers originating from a contig that contains a good marker in one of the HAPPY linkage groups. The good markers are also situated at the ends of HAPPY linkage groups. Small contigs (unmapped in this study) are believed to lie in the gaps between contigs in the map, based on what was observed in the BACclone integration.

Because the HAPPY map is limited to a range of about $100 \mathrm{~kb}$ (due to the selected fragment size of DNA), marker-pairs from the ends of contigs longer than this are not linked by the HAPPY data, but are of course joined by the contig itself. Contig information was thus integrated to help further improve the map. Although the 2010 assembly contains larger contigs, we find conflicts between

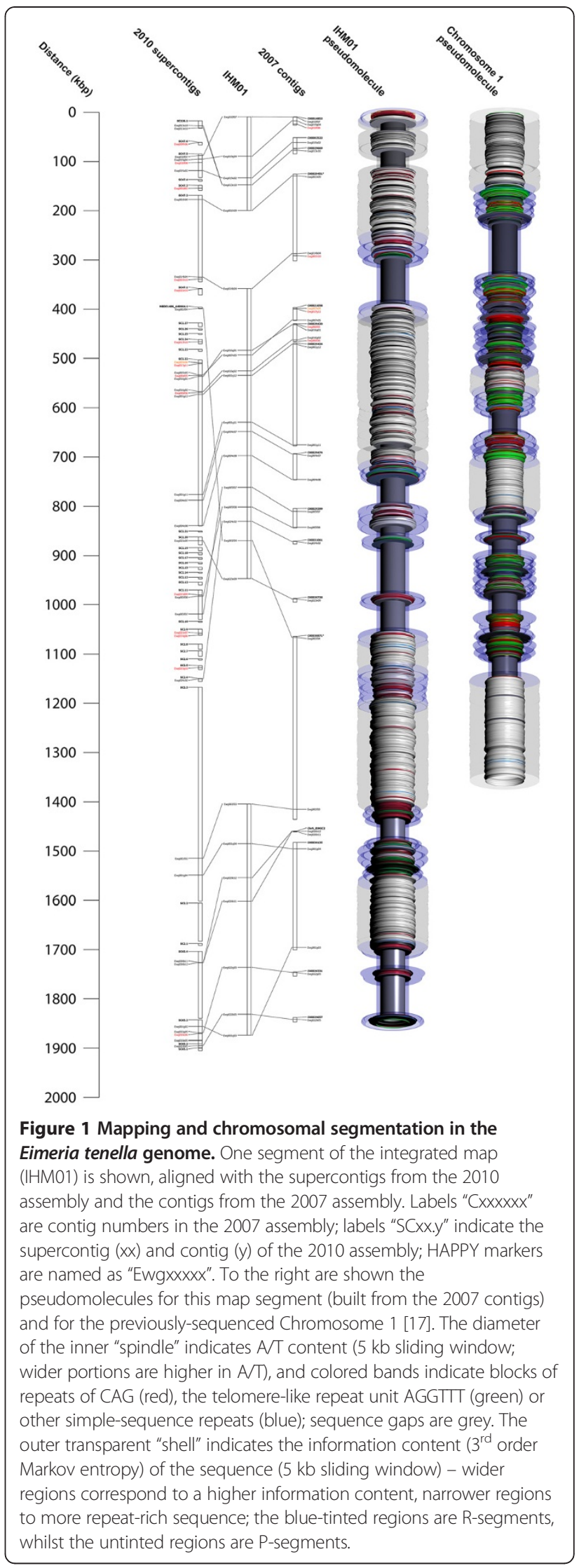


the assembly with HAPPY map (alignment between the integrated map and both of the assemblies is shown in Figure 1 and Additional file 4) and BAC linkage data. Therefore, we utilize contigs from the 2007 assembly for this purpose. Twenty pairs of markers are linked in this way. After integration, an additional nine linkages between linkage groups and 10 linkages to singletons were added to the map (details in Additional file 2).

\section{Simultaneous validation of contig quality and HAPPY map reliability}

To monitor the quality and reliability of the HAPPY map, markers were designed at $20 \mathrm{~kb}$ intervals along the two largest contigs of the draft assembly. All the good markers from these two contigs were used in the subsequent map-making process and were successfully arranged into two linkage groups that correspond to their respective contigs (details in Additional file 5), and the HAPPY map arrangement of these markers corresponds well with their locations in the contigs. Similarly encouraging results come from HAPPY markers designed at opposite ends of contigs smaller than $100 \mathrm{~kb}$ (the maximum range of the HAPPY map): in all cases, these pairs of markers are linked, and the distance between them as estimated from the HAPPY data corresponds well with the contig length.

The BAC-end sequences also suggested that HAPPY mapping has correctly ordered the contigs, as most of the HAPPY linkages are supported by at least one BAC clone. All these results strongly suggest that both the HAPPY data and the 2007 assembly are of good quality.

\section{Chromosomal assignments of HAPPY linkage groups}

Fifteen chromosomally-assigned genes representing ten chromosomes were used to help assign linkage groups to chromosomes (Table 1). Eleven of these genes provided good markers and, of these, ten showed linkage to other markers and were incorporated into the map. Together with the position information of the genes in the current assembly, we have successfully assigned eight linkage groups to eight different chromosomes. Among them, the largest linkage group was found to belong to Chromosome 9. None of the chromosomes is fully covered by linkage groups, but in most cases a small number of linkage groups represent each chromosome (details in Tables 1 and 2).

\section{The integrated HAPPY map}

As a result of incorporating BAC-end and contig sequences, an integrated HAPPY map was constructed [Figure 1; Additional file 4]. The map consists of 59 linkage groups that range from $100 \mathrm{~kb}$ to $1.7 \mathrm{Mb}$ in size, covering $\sim 31.0 \mathrm{Mb}$. Eight of these groups are chromosomally assigned.

\section{Segmentation of the genome into P- and R-regions}

We also examined whether the segmentation of the genome into feature-rich ("R") and feature-poor ("P")

Table 1 Details of chromosomally-assigned genes

\begin{tabular}{|c|c|c|c|c|c|c|}
\hline Chr & Gene & Reference & Gene size (bp) & GenBank accession no. & Contig aligned & Gene HAPPY marker \\
\hline 3 & EtMIC3 & {$[30]$} & 3680 & FJ374765 & contig_00031447 & NS \\
\hline \multirow[t]{2}{*}{4} & SAG 19 & * & 816 & AJ586544 & contig_00017931 & S \\
\hline & & & & & contig_00031465 & \\
\hline 4 & SAG 20 & * & 816 & AJ586549 & contig_00031465 & S \\
\hline 5 & EtMIC4 & {$[30]$} & 7766 & AJ306453 & contig_00029206 & S \\
\hline 6 or 7 & SAG 15 & * & 792 & AJ586550 & contig_00031359 & S \\
\hline 6 or 7 & SAG 18 & * & 807 & AJ586548 & contig_00031359 & S \\
\hline 9 & EtMIC2 & {$[30]$} & 1957 & Z71755 & contig_00030135 & s \\
\hline 9 & EtMIC5 & {$[30]$} & 3334 & AJ245536 & contig_00010048 & NS \\
\hline 9 or 10 & SAG 5 & * & 774 & AJ586532 & contig_00030134 & S \\
\hline 9 or 10 & SAG 7 & $*$ & 762 & AJ586533 & contig_00030134 & S \\
\hline \multirow[t]{2}{*}{10} & $5 \mathrm{~S}$ rRNA & [31] & 728 & M86547 & contig_00001191 & NS \\
\hline & & & & & contig_00012636 & \\
\hline 11 & SAG 1 & * & 762 & AJ586531 & contig_00031646 & S \\
\hline 11 & SAG 2 & * & 813 & AJ586540 & contig_00031646 & S \\
\hline 12 & 18 S-5.8 S-28S_rDNA & {$[31]$} & 1286 & AY779514 & contig_00004986 & NS \\
\hline 13 & EtMIC1 & {$[30]$} & 5990 & AF032905 & contig_00029639 & S \\
\hline
\end{tabular}

*Tabares $\mathrm{E}$ and Tomley F, unpublished.

$S$ - Successfully mapped.

NS - Not successfully mapped. 
Table 2 Location of chromosomally-assigned genes or markers

\begin{tabular}{cccc}
\hline Chr & $\begin{array}{c}\text { Chromosome size* } \\
(\mathbf{M b})\end{array}$ & $\begin{array}{c}\text { Linkage } \\
\text { group }\end{array}$ & $\begin{array}{c}\text { Linkage group size } \\
\text { (Mb) }\end{array}$ \\
\hline 3 & $\sim 2$ & IHM13 & 0.68 \\
4 & $\sim 2$ & HM07 & 1.00 \\
5 & $\sim 2.5$ & IHM04 & 1.40 \\
6 or 7 & $\sim 3$ & IHM14 & 0.62 \\
9 & $\sim 4$ & IHM01 & 1.70 \\
9 or & $\sim 4$ & IHM09 & 0.90 \\
10 & & & \\
10 & $\sim 4$ & Not identified & - \\
11 & $\sim 4.5$ & IHM20 & 0.56 \\
12 & $\sim 5$ & Not identified & - \\
13 & $\sim 6$ & IHM49 & 0.23 \\
\hline
\end{tabular}

*Based on PFGE of the Eimeria tenella genome [32].

segments, as noted on Chromosome 1 [17], could be seen on the whole genome map. An algorithm (see Methods) was used to partition larger contigs into P- and R-regions, and to identify smaller contigs as either $\mathrm{P}$ or $\mathrm{R}$, based on their content of simple-sequence repeats. The overall pattern of large, alternating P- and R-segments seems to be preserved throughout the genome [Figure 1; Figure 2], with about three or four R-segments per $\mathrm{Mb}$, interspersed with P-segments of roughly similar size. Deviations from this pattern are probably due to the difficulty in accurately assigning small contigs as $\mathrm{P}$ or $\mathrm{R}$, and in defining $\mathrm{P} / \mathrm{R}$ boundaries which fall close to the ends of contigs. Most of the linkage groups and most of the larger sequence contigs end in R-regions, consistent with the expectation that most of the unmapped and unassembled parts of the genome are repeat-rich R-type sequence.
From earlier analysis of Chromosome 1 [17] and from analysis of markers on the largest two contigs of the 2007 assembly, it is obvious that P-regions have a higher density of good markers than R-regions [Figure 1; Figure 2]. This is due largely to the non-unique nature of much of the R-region sequence (leading to multi-copy markers), and probably also to abundant simple-sequence repeats, which we find interfere with amplification of adjoining non-repetitive sequence.

The 661 HAPPY markers which fall into linkage groups cover $\sim 31.0 \mathrm{Mb}$ of the genome, giving an average spacing on mapped regions of $\sim 46.9 \mathrm{~kb}$. There are 105 singletons (unlinked HAPPY markers) in the integrated map. Assuming that these are distributed evenly across the unmapped areas, their average spacing is approximately $276 \mathrm{~kb}$. This is more than double the range of the mapping panel $(\sim 100 \mathrm{~kb})$, explaining why they do not link to other markers.

\section{Copy-number variation between strains of $E$. tenella}

Although HAPPY mapping reveals an abundance of multi-copy sequences (which were represented only once in the assembly), the exact copy-numbers of these sequences in the genome is still unknown. In humans, multi-copy regions tend to vary in copy-number between individuals [25]. Furthermore, structural variations have never been studied in the E. tenella genome. Hence, we set out to investigate the HAPPY markers for variation in copy-number between strains of $E$. tenella using the molecular copy-number counting (MCC) method. We randomly selected 48 of the HAPPY markers for this analysis.

Of 48 markers tested on the reference Houghton $(\mathrm{H})$ strain, one marker failed to amplify for unknown reasons.
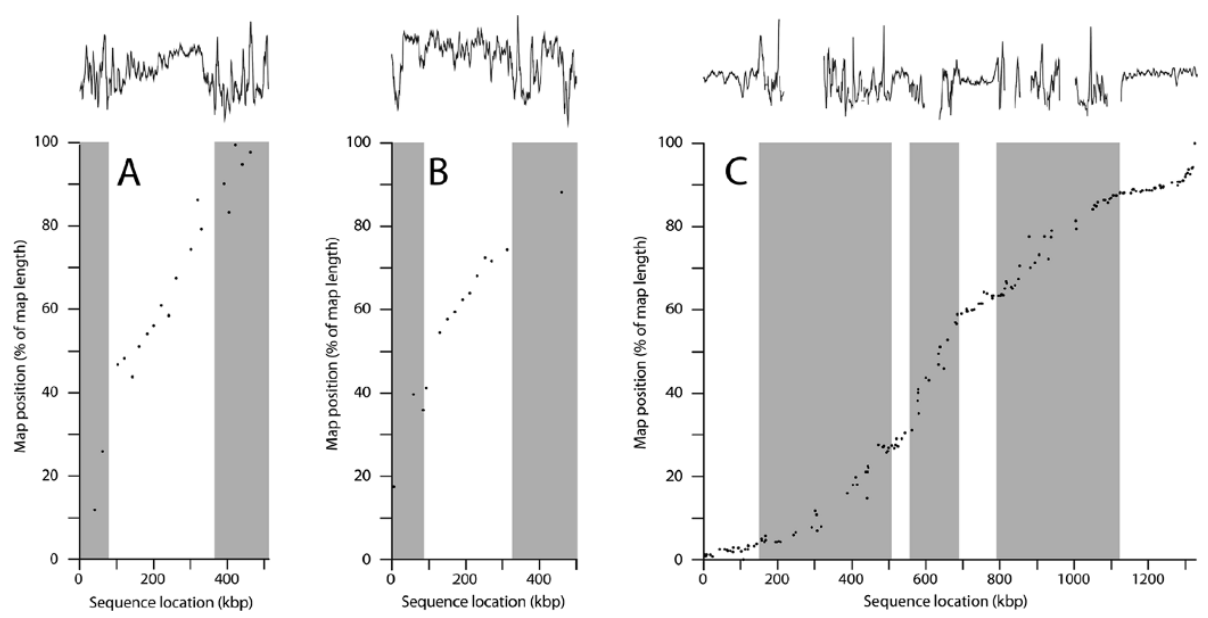

Figure 2 Comparison between assembled sequence and map data. Each graph shows the positions of mapped markers on the map, plotted against their positions in the assembled sequence, for the two largest sequence contigs of the 2007 assembly $(\mathbf{A}, \mathbf{B})$ and for the previously-reported Chromosome 1 sequence (C; 17$])$. Grey bars indicate R-regions. Above each graph is plotted the AT content (5 kb sliding window) of the sequence. 
Twelve markers (25\%) were detected to be present in multiple copies (relative copy-number $>1.5$ ) in the genome [Figure 3]. Four of these twelve markers are present in more than two copies per haploid genome.

The analysis carried out on the Weybridge (Wey) strain also revealed eleven of the markers to be multicopy while analysis on the Wisconsin (Wis) strain showed only nine multi-copy markers. Although the Wey strain had almost the same number of multi-copy markers as the $\mathrm{H}$ strain, most of them were lower in copy-number. The Wis strain has the lowest number of multi-copy markers but one of them (marker 44) is present in triple the normal copy-number. One marker, marker 27, did not show amplification in the Wis strain.

Overall, four markers were multi-copy in all three strains while sixteen markers were found to have differences in copy-number between the strains. We therefore estimate that, within any one strain, about $20 \%$ of sequences (other than short tandem repeats) are present in multiple copies $(11,12$ and 9 out of the 47 markers, in the three strains analyzed), if our selection of markers is representative. Moreover, about a third of sequences (again, assuming our markers to be representative) differ in copy-number between strains. Further work is needed to clarify the extent of the duplicated sequence blocks, and their possible relation to genes.

\section{Discussion}

The analysis of the Chromosome 1 sequence [17] showed a striking segmentation, with feature-poor (P) regions alternating with feature-rich $(\mathrm{R})$ regions. The $\mathrm{P}$ regions are slightly longer, have a higher and more uniform AT content than the R-regions and are almost free of simple repeats. In contrast, R-regions contain an abundance of simple repeats including tandemly repeated trinucleotides, the telomere-like AGGGTTT heptamer, the TGCATGCA palindromic octamer (which seems to be peculiar to a subset of apicomplexans; [26]), and also LINE transposons. It was speculated that the rest of the genome of E. tenella was organized in a similar segmented fashion.

The integrated map described here has revealed the structure for about half of the E. tenella genome based on the relative physical size of the map in comparison with the estimated genome size $(60 \mathrm{Mb})$. Our analysis suggests that this half consists mainly of P-type sequence, largely because markers in R-regions are usually multicopy and therefore unmappable. If most R-regions are larger than $140 \mathrm{~kb}$ (the smallest R-region on Chromosome 1), then this would explain the inability of the map to connect across most $\mathrm{R}$ regions by means of HAPPY linkage or BAC clones.

Regarding the accuracy of the integrated map, we do see some conflicts in marker order when compared with the sequence assembly (see, for example, Additional file $4)$. For the most part, these are local inversions in line with the expected resolution of the HAPPY map; others, particularly where they involve small isolated sequence contigs, are likely to represent errors in the sequence assembly. As with all complex genomes, mapping and sequencing are iterative and interdependent processes, and there is no universally agreed metric for measuring the goodness of a map, nor agreement on how to weight common local errors against rarer large-scale errors. However, it is generally agreed that maps which integrate several datasets (in this case, HAPPY data, BACend data and contig data) are more robust than those which depend on a single method.

If we are correct in assuming that most of the unmappable multicopy markers originate from R-regions, this suggests that the $\mathrm{R}$-regions are rich in repeated sequences (in addition to the simple-sequence repeats which are avoided in marker selection), and that a segment from an R-region on one chromosome may be duplicated at an R-region of another chromosome. About $20 \%$ of genome may be repeated in this way, in addition to the $14 \%$ made up of simple-sequence repeats (based on Chromosomes 1 and 2). This may account for the fact that the draft sequence assembly represents only about $78 \%$ of the genome.

Comparisons between E. tenella strains are scarce but have generally shown moderate variation at the sequence level. For instance, the recent comparison of the $\sim 9 \mathrm{~kb}$ glucose-6-phosphate isomerase genomic locus between the $\mathrm{H}$, Wis and Wey strains revealed 33 SNPs and 14 indels [19], or a variation of around half of one percent of nucleotides. In contrast, our preliminary study based on CNV analysis showed the possibility of much more widespread structural variation between these three strains.

\section{Conclusions}

The integrated HAPPY map has revealed the probable structure of the E. tenella genome, and explains why the ongoing sequencing program has encountered difficulties. It suggests that the genome is architectured segmentally, alternating between P- and R-regions, with an average of about four or five P-regions per $\mathrm{Mb}$. Rregions are likely to contain tracts of repeated sequence amounting to $>20 \%$ of the genome, as well as a further $14 \%$ of simple-sequence repeats. This segmental structure and the repetitive nature of the R-segments probably explain both the gaps in the integrated map and the incompleteness of the draft sequence assembly.

There are also indications that much of the genome displays copy-number variation between $E$. tenella strains. Given that parasites must constantly adapt to oppose emerging resistance in the host, it is tempting to 


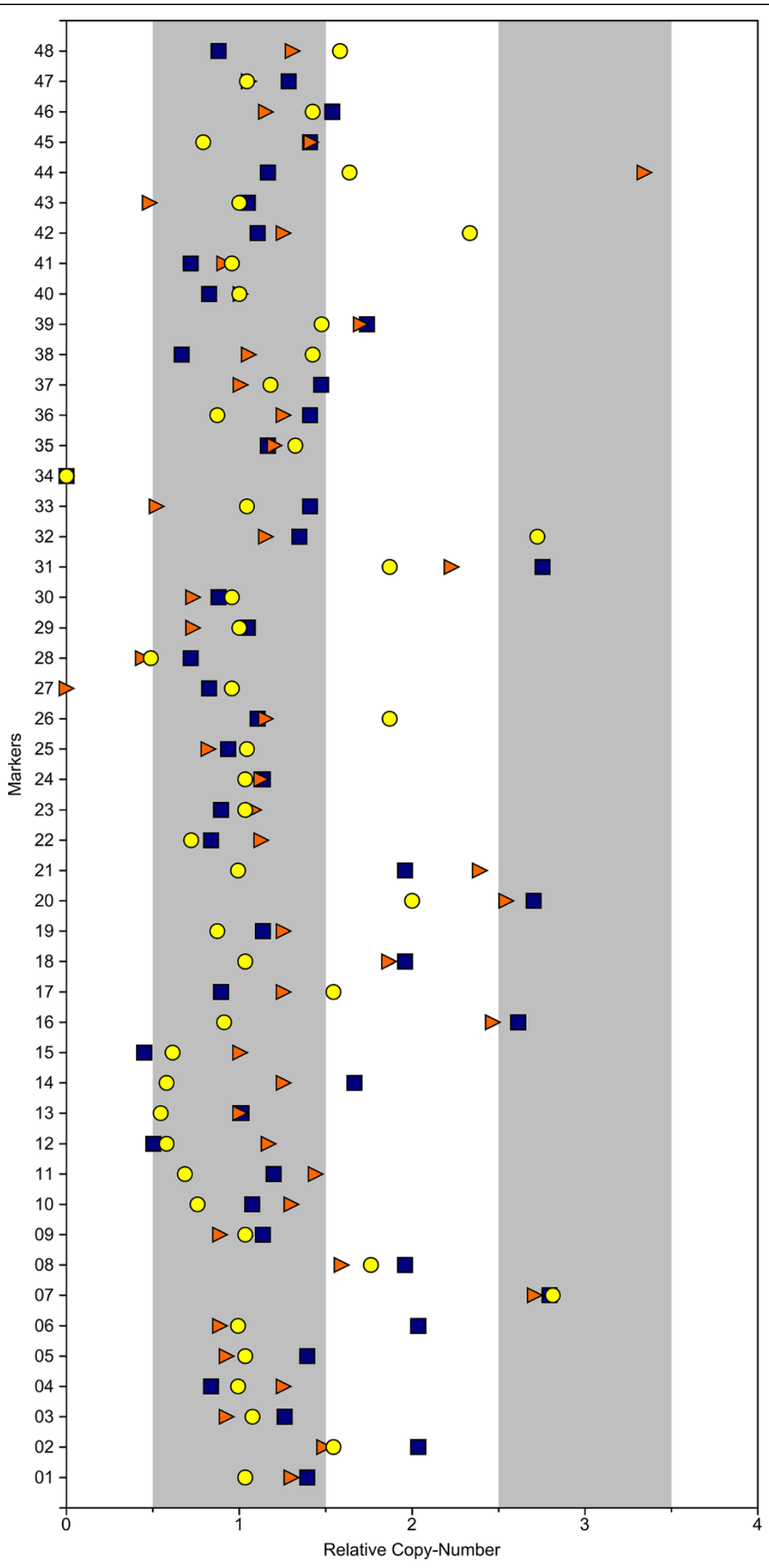

Figure 3 Copy-number variations between Eimeria tenella strains. For 48 markers (numbered at left) relative copy-number was measured on the Houghton (blue squares), Wisconsin (red triangles) and Weybridge (yellow circles) strains, as described in the text. 
speculate that the segmental architecture contributes to a structurally dynamic genome, lubricated by the repeatrich R-regions. This, in turn, may play an important role in the rapid emergence of drug resistance which is known to be a feature of Eimeria.

\section{Methods HAPPY mapping}

The HAPPY mapping was performed essentially as described previously [26-28] on a mapping panel containing fragments selected at approximately $100 \mathrm{~kb}$, with customization in the primer design and marker selection process. The draft genome assembly contigs were divided into fragments of $\sim 2 \mathrm{~kb}$ in length. All of these $2 \mathrm{~kb}$ fragments were subjected to primer design using UniversalPrimerDesigner (Paul H. Dear, unpublished). This software was set to find the optimum set of heminested primers (forward external, forward internal and reverse) for each $2 \mathrm{~kb}$ fragment. The designed primers had a predicted melting temperature of $55-62^{\circ} \mathrm{C}$, with two $\mathrm{G}$ or $\mathrm{C}$ nucleotides at the 3 ' end and one $\mathrm{G}$ or $\mathrm{C}$ nucleotide at the 5' end, and length 18-22 bases. Each set of primers was designed to produce an internal amplicon of 100-200 bp (external amplicon length 150$350 \mathrm{bp}$ ) with an $\mathrm{A}+\mathrm{T}$ content not exceeding $80 \%$. Contigs similar to the Chromosome 1 and 2 sequences were filtered. The uniqueness of each primer set was then checked against the genome assembly. Markers were picked at contig ends for contigs larger than $17 \mathrm{~kb}$, and one marker for contigs smaller than $17 \mathrm{~kb}$. Where markers failed (for example, were found to be multi-copy), further markers were chosen from nearby sequences in an attempt to find successful markers.

\section{$\mathrm{P} / \mathrm{R}$ region assignment}

Contigs larger than $5 \mathrm{~kb}$ were divided into P- and Rsegments using custom software, (Paul H. Dear, unpublished). Briefly, all of the simple-sequence repeats (of [CAG]n or [AGGGTTT]n, where $n \geq 3$ ) which pepper the R-regions were identified, and both they and any intervening stretches of sequence shorter than $4 \mathrm{~kb}$ were marked as putative R-segments, with the remainder (stretches of $>4 \mathrm{~kb}$ lacking simple-sequence repeats) being marked as putative P-segments. Based on analysis of Chromosomes 1 and 2, we assumed that genuine Por R-segments would each be larger than $30 \mathrm{~kb}$. Therefore, the shortest putative P- or R-segment was eliminated (so that, for example, a $5 \mathrm{~kb}$ putative R-region flanked by larger P-regions would be merged to become a single contiguous P-region); this was done iteratively until no segment smaller than $30 \mathrm{~kb}$ remained, apart from the first and last segments of the contig.

Contigs smaller than $5 \mathrm{~kb}$ were not divided into segments, but were instead classified as entirely P-type, R- type or unclassifiable based on their overall content of simple-sequence motifs (less than 0.5 motifs, more than 1 motif, or between 0.5 and 1 motifs per kilobase, respectively).

The parameters for this analysis are somewhat arbitrary, but were chosen such that they accurately identified the $\mathrm{P} / \mathrm{R}$ segmentation that had been previously noted for Chromosomes 1 and 2. Moreover, slight variation in these parameters, or the use of analyses based on sequence information content and base composition (which also distinguish P- from R-segments) gave essentially similar results (not shown).

\section{Data integration}

BAC-end sequences were mapped to the draft genome using ssahaEST [29] then processed to remove unpaired sequences and sequences that did not map uniquely to a single region. For the chromosomal assignment of map segments, HAPPY markers were designed for fifteen chromosomally-assigned genes and mapped as described.

\section{Copy-number variation analysis}

Forty-eight randomly selected markers (as described in primer design) were typed on panels of sub-genomic aliquots of sheared DNA from three different E. tenella strains (Houghton, Weybridge and Wisconsin) using the MCC approach [21]. Panels of 96 aliquots containing approximately 0.3 genomes of sheared DNA were constructed for each strain to detect up to 3-fold change in copy-number variation. The selected markers were then typed using similar hemi-nested PCR following the same protocol as for the HAPPY mapping. The proportion of aliquots positive for any marker allows one to calculate its abundance in the panel (Poisson distribution), and hence its copy-number relative to other markers. Further details are given in reference [21].

\section{Additional files}

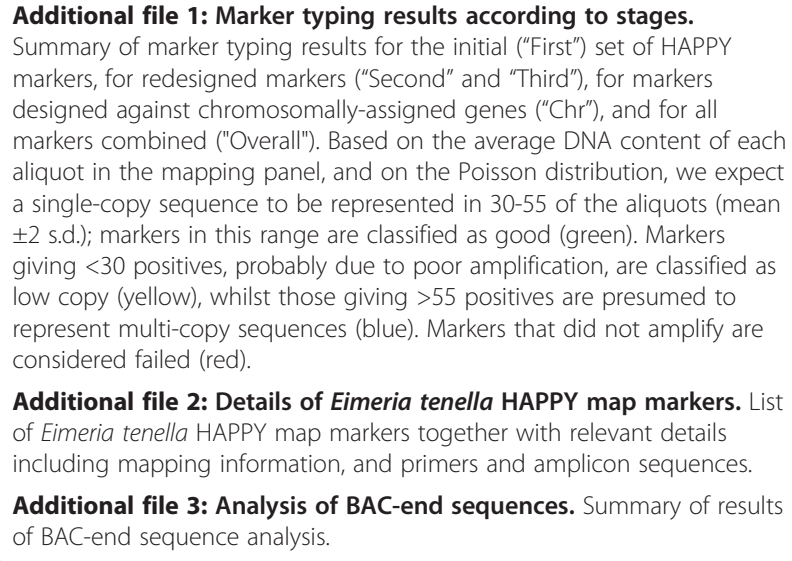

Additional file 2: Details of Eimeria tenella HAPPY map markers. List of Eimeria tenella HAPPY map markers together with relevant details including mapping information, and primers and amplicon sequences.

Additional file 3: Analysis of BAC-end sequences. Summary of results of BAC-end sequence analysis. 
Additional file 4: Alignment of the integrated HAPPY map with WGS assemblies of the E. tenella genome. Graphical representation of the alignment of the HAPPY map and sequence of the Eimeria tenella draft genome assembly contigs. For each map segment, labels "Cxxxxxxxx" are contig numbers in the 2007 assembly; labels "SCXX.y" indicate the supercontig (xx) and contig (y) of the 2010 assembly; HAPPY markers are named as "Ewgxxxxxx"; asterisks in 2010 assembly denote markers that mapped to more than one locus in the assembly.

Additional file 5: The comparison of the HAPPY map and sequence of (A) contig_00031646 and (B) contig_00031359. Graphical

representation of the alignment of the HAPPY map and sequence of the two largest Eimeria tenella draft genome assembly contigs.

\section{Competing interests}

The authors declare that they have no competing interests.

\section{Authors' contributions}

K-LW and PHD conceptualized the research plan. L-SL constructed the HAPPY map, and together with Y-LT, HA and PHD analyzed the data. L-SL drafted the manuscript. K-LW and PHD critically revised the manuscript. K-LW and PHD supervised and coordinated the study. All authors read and approved the final manuscript.

\section{Acknowledgements}

We wish to thank Martin Shirley and Damer Blake for parasite materials, Enrique Tabares and Fiona Tomley for unpublished data, and Arnab Pain of the Eimeria tenella genome project at the Wellcome Trust Sanger Institute for making the latest genome assembly and BAC-end sequences available for use in this study. This work was supported by the Genomics and Molecular Biology Initiatives Programme of the Malaysia Genome Institute, Ministry of Science, Technology and Innovation Malaysia (grant number 07-05-16-MGIGMB10).

\section{Author details}

${ }^{1}$ School of Biosciences and Biotechnology, Faculty of Science and Technology, Universiti Kebangsaan Malaysia, 43600, UKM Bangi, Selangor DE, Malaysia. ${ }^{2}$ Malaysia Genome Institute, Jalan Bangi, 43000, Kajang, Selangor DE, Malaysia. ${ }^{3}$ MRC Laboratory of Molecular Biology, Hills Road, Cambridge CB2 OQH, United Kingdom.

Received: 17 February 2012 Accepted: 1 August 2012 Published: 13 August 2012

\section{References}

1. Gardner MJ, Hall N, Fung E, White O, Berriman M, Hyman RW, Carlton JM, Pain A, Nelson KE, Bowman S, Paulsen IT, James K, Eisen JA, Rutherford K, Salzberg SL, Craig A, Kyes S, Chan MS, Nene V, Shallom SJ, Suh B, Peterson J, Angiuoli S, Pertea M, Allen J, Selengut J, Haft D, Mather MW, Vaidya AB, Martin DM, et al: Genome sequence of the human malaria parasite Plasmodium falciparum. Nature 2002, 419:498-511.

2. Abrahamsen MS, Templeton TJ, Enomoto S, Abrahante JE, Zhu G, Lancto CA, Deng M, Liu C, Widmer G, Tzipori S, Buck GA, Xu P, Bankier AT, Dear PH, Konfortov BA, Spriggs HF, lyer L, Anantharaman V, Aravind L, Kapur V: Complete genome sequence of the apicomplexan, Cryptosporidium parvum. Science 2004, 304:441-445.

3. Gardner MJ, Bishop R, Shah T, de Villiers EP, Carlton JM, Hall N, Ren Q, Paulsen IT, Pain A, Berriman M, Wilson RJ, Sato S, Ralph SA, Mann DJ, Xiong Z, Shallom SJ, Weidman J, Jiang L, Lynn J, Weaver B, Shoaibi A, Domingo AR, Wasawo D, Crabtree J, Wortman JR, Haas B, Angiuoli SV, Creasy TH, Lu C, Suh B, et al: Genome sequence of Theileria parva, a bovine pathogen that transforms lymphocytes. Science 2005, 309:134-137.

4. Khan A, Böhme U, Kelly KA, Adlem E, Brooks K, Simmonds M, Mungall K, Quail MA, Arrowsmith C, Chillingworth T, Churcher C, Harris D, Collins M, Fosker N, Fraser A, Hance Z, Jagels K, Moule S, Murphy L, O'Neil S, Rajandream MA, Saunders D, Seeger K, Whitehead S, Mayr T, Xuan X, Watanabe J, Suzuki Y, Wakaguri H, Sugano $S$, et al: Common inheritance of chromosome la associated with clonal expansion of Toxoplasma gondii. Genome Res 2006, 16:1119-1125.

5. Brayton KA, Lau AO, Herndon DR, Hannick L, Kappmeyer LS, Berens SJ, Bidwell SL, Brown WC, Crabtree J, Fadrosh D, Feldblum T, Forberger HA,
Haas BJ, Howell JM, Khouri H, Koo H, Mann DJ, Norimine J, Paulsen IT, Radune D, Ren Q, Smith RK Jr, Suarez CE, White O, Wortman JR, Knowles DP $\mathrm{Jr}$, McElwain TF, Nene VM: Genome sequence of Babesia bovis and comparative analysis of apicomplexan hemoprotozoa. PLoS Pathog 2007, 3:e148.

6. Shirley MW: Research on avian coccidia: an update. Br Vet J 1992, 148:479-499.

7. Belli Sl, Smith NC, Ferguson DJP: The coccidian oocyst: a tough nut to crack! Trends Parasitol 2006, 22:416-423.

8. Chapman HD, Shirley MW: The Houghton strain of Eimeria tenella: a review of the type strain selected for genome sequencing. Avian Pathol 2003, 32:115-127.

9. Shirley MW, Smith AL, Tomley FM: The biology of avian Eimeria with an emphasis on their control by vaccination. Adv Parasitol 2005, 60:285-330.

10. Shirley MW, Ivens A, Gruber A, Madeira AMBN, Wan K-L, Dear PH, Tomley FM: The Eimeria genome projects: a sequence of events. Trends Parasitol 2004, 20:199-201.

11. Green ED: Strategies for the systematic sequencing of complex genomes. Nat Rev Genet 2001, 2:573-583.

12. Beyer A, Bandyopadhyay S, Idekar T: Integrating physical and genetics maps: from genomes to interaction networks. Nat Rev Genet 2007, 8:699-710.

13. Lewin HA, Larkin DM, Pontius J, O'Brien SJ: Every genome sequence needs a good map. Genome Res 2009, 19:1925-1928.

14. Shirley MW, Harvey DA: A genetic linkage map of the apicomplexan protozoan parasite Eimeria tenella. Genome Res 2000, 10:1587-1593.

15. Hoogendoorn B, Toye AA, Bumstead N, Shirley M: Mapping the genome of Eimeria tenella: use of yeast artificial chromosome library as the basis of a physical map. Parasitol Res 1998, 84:13-16.

16. Dear PH, Cook PR: HAPPY mapping: linkage mapping using a physical analogue of meiosis. Nucl Acids Res 1993, 21:13-20.

17. Ling $\mathrm{K}-\mathrm{H}$, Rajandream MA, Rivailler P, Ivens A, Yap S-J, Madeira AM, Mungall K, Billington K, Yee W-Y, Bankier AT, Carroll F, Durham AM, Peters N, Loo S-S, Isa MN, Novaes J, Quail M, Rosli R, Nor Shamsudin M, Sobreira TJ, Tivey AR, Wai S-F, White S, Wu X, Kerhornou A, Blake D, Mohamed R, Shirley M, Gruber A, Berriman M, et al: Sequencing and analysis of chromosome 1 of Eimeria tenella reveals a unique segmental organization. Genome Res 2007, 17:311-319.

18. Barta JR, Coles BA, Schito ML, Fernando MA, Martin A, Danforth DH: Analysis of infraspecific variation among five strains of Eimeria maxima from North America. Int J Parasitol 1998, 28:485-492.

19. Loo S-S, Blake DP, Mohd-Adnan A, Mohamed R, Wan K-L: Eimeria tenella glucose-6-phosphate isomerase: molecular characterization and assessment as a target for anti-coccidial control. Parasitol 2010, 137:1169-1177.

20. Freeman JL, Perry GH, Feuk L, Redon R, McCarroll SA, Altshuler DM, Aburatani H, Jones KW, Tyler-Smith C, Hurles ME, Carter NP, Scherer SW, Lee C: Copy number variation: new insight in genome diversity. Genome Res 2006, 16:949-961.

21. Daser A, Thangavelu M, Pannell R, Forster A, Sparrow L, Chung G, Dear PH, Rabbitts TH: Interrogation of genomes by molecular copy-number counting (MCC). Nat Methods 2006, 3:447-453. Erratum in: Nat Methods 2006, 3: 579

22. McCaughan F, Darai-Ramqvist E, Bankier AT, Konfortov BA, Foster N, George PJ, Rabbitts TH, Kost-Alimova M, Rabbitts PH, Dear PH: Microdissection molecular copy-number counting ( $\mu \mathrm{MCC}$ ) - unlocking cancer archives with digital PCR. J Pathol 2008, 216:307-316.

23. McCaughan F: Molecular copy-number counting: potential of singlemolecule diagnostics. Expert Rev Mol Diagn 2009, 9:309-312.

24. McCaughan F, Pole JC, Bankier AT, Konfortov BA, Carroll B, Falzon M, Rabbitts TH, George PJ, Dear PH, Rabbitts PH: Progressive 3q amplification consistently targets SOX2 in preinvasive squamous lung cancer. Am J Respir Crit Care Med 2010, 182:83-91.

25. Sharp AJ, Locke DP, McGrath SD, Cheng Z, Bailey JA, Vallente RU, Pertz LM, Clark RA, Schwartz S, Segraves R, Oseroff W, Albertson DG, Pinkel D, Eichler EE: Segmental duplications and copy-number variation in human genome. Am J Hum Genet 2005, 77:78-88.

26. Bankier AT, Spriggs HF, Fartmann B, Konfortov BA, Madera M, Vogel C Teichmann SA, Ivens A, Dear PH: Integrated mapping, chromosomal sequencing and sequence analysis of Cryptosporidium parvum. Genome Res 2003, 13:1787-1799. Erratum in: Genome Res 2004, 14: 327. 
27. Konfortov BA, Cohen HM, Bankier AT, Dear PH: A high-resolution HAPPY map of Dictyostelium discoideum chromosome 6. Genome Res 2000, 10:1737-1742

28. Glöckner G, Eichinger L, Szafranski K, Pachebat JA, Bankier AT, Dear PH, Lehmann R, Baumgart C, Parra G, Abril JF, Guigó R, Kumpf K, Tunggal B, Cox E, Quail MA, Platzer M, Rosenthal A, Noegel AA: Dictyostelium Genome Sequencing Consortium: Sequence and analysis of chromosome 2 of Dictyostelium discoideum. Nature 2002, 418:79-85.

29. ssahaEST; http://www.sanger.ac.uk/resources/software/ssahaest/.

30. Ryan R, Shirley M, Tomley F: Mapping and expression of microneme genes in Eimeria tenella. Intl J Parasitol 2000, 30:1439-1499.

31. Shirley MW: The genome of Eimeria spp., with special reference to Eimeria tenella - a coccidium from the chicken. Int J Parasitol 2000 30:485-493.

32. Shirley MW: The genome of Eimeria tenella: further studies on its molecular organization. Parasitol Res 1994, 80:366-373.

\section{Submit your next manuscript to BioMed Central and take full advantage of:}

- Convenient online submission

- Thorough peer review

- No space constraints or color figure charges

- Immediate publication on acceptance

- Inclusion in PubMed, CAS, Scopus and Google Scholar

- Research which is freely available for redistribution 